\title{
A clinical, microbiological and economic analysis of a national service for the rapid molecular diagnosis of tuberculosis and rifampicin resistance in Mycobacterium tuberculosis
}

\author{
F. A. DROBNIEWSKI, S. A. WATTERSON, S. M. WILSON and G. S. HARRIS
}

Public Health Laboratory Service Mycobacterium Reference Unit, Dulwich Public Health Laboratory and Department of Microbiology, Guy's King's and St Thomas' School of Medicine, London SE22 8QF

A clinical, microbiological and economic study of a national rapid molecular service for the identification of Mycobacterium tuberculosis and the determination of rifampicin resistance in smear-positive sputum samples (and other primary specimens) was performed. Ninety-one primary specimens, of which 55 were smear-positive sputum, were examined by molecular and conventional assays. Concordance of molecular results from smear-positive sputum specimens with tuberculosis diagnosis and rifampicin resistance by conventional analysis was $52(94.5 \%)$ of 55 and $44(91.7 \%)$ of 48 , respectively. Concordance of molecular analysis on all primary specimens was 81 $(89.0 \%$ ) of 91 (diagnosis) and 55 (90.2\% ) of 61 (rifampicin resistance). Approximately 28 days were saved in the time to diagnosis by using the molecular assay. Hospitals can reduce the cost of inappropriate isolation of patients with risk factors for multiple drugresistant tuberculosis (MDRTB) who subsequently are shown to have drug-sensitive tuberculosis. At one hospital potential annual savings were between $£ 50000$ and f150000. Of the nine MDRTB cases identified, all had a previous diagnosis of tuberculosis, $78 \%$ were born overseas, $44 \%$ were known to be non-compliant with therapy, but only one case $(12.5 \%)$ was HIV positive. HIV status was not significantly different between MDRTB and drug-sensitive tuberculosis cases. Over $75 \%$ of specimens were taken while the patient was on therapy. I solates from $>50 \%$ of the MDRTB cases were resistant to three or more drugs and one was resistant to seven drugs. All patients were placed on additional therapy once the molecular result was known; this was subsequently modified based on the results of in-vitro drug susceptibility testing. All survived at least 6 months of follow-up. There was no difference in the proportion of successful cultures from smear-positive samples from patients with drug-sensitive tuberculosis or MDRTB who were on therapy. Molecular rifampicin resistance assays are reliable for diagnosis in cases with smear-positive disease.

\section{Introduction}

Infection with Mycobacterium tuberculosis causes nearly 8 million new cases of tuberculosis and 3 million deaths annually [1]. The worldwide extent of drug resistance is not clear, but resistance to at least one drug has been reported in nearly $10 \%$ of patients with no prior treatment; the prevalence of cases of primary isoniazid resistance, rifampicin resistance and multiple drug-resistant tuberculosis (MDRTB, i.e., cases due to strains resistant to at least isoniazid and

Received 19 July 1999; accepted 1 Sept. 1999.

Corresponding author: Dr F. A. Drobniewski. rifampicin) was $7.3 \%, 1.8 \%$ and $1.4 \%$, respectively, in one study [2].

Drug-resistant $M$. tuberculosis, and particularly MDRTB, compromises clinical success and the effectiveness of tuberculosis control programmes because of the prolonged infectivity of the patients $[3,4]$. Dramatic outbreaks of MDRTB, particularly in HIVinfected patients, in the USA $[5,6]$ and subsequently in Europe [7] focused international concern on the major public health threat posed by drug-resistant tuberculosis. High case-fatality rates (approaching 90\% in HIV-positive individuals) were reported from the USA $[3,4,8]$. 
The UK is a low-incidence country for tuberculosis (c. 6000 new cases per annum; notification rate of $c$. $10 / 100000$ population), but the incidence of both drug-sensitive and -resistant tuberculosis is highly polarised geographically $[9,10]$. London, for example, has a tuberculosis notification rate almost four times the national average [9]. From 1993 to 1996, cases of initial MDRTB (i.e., patients infected with MDRTB isolates who are believed not to have had a diagnosis of tuberculosis or prior therapy) increased from $0.6 \%$ to $1.6 \%[10]$.

Significant advances have been made in understanding the genetic basis of drug resistance, particularly for rifampicin; in c. $95 \%$ of rifampicin-resistant isolates, resistance is due to mutations in an 81-bp region of the rpoB gene [11]. Therefore, this gene is a good target for molecular genotypic diagnostic methods [12-17]. In the UK, $>90 \%$ of rifampicin-resistant isolates are also resistant to isoniazid, which makes rifampicin resistance a useful surrogate marker for MDRTB.

Other studies have described the technical performance of molecular identification assays for M. tuberculosis, but there has been little analysis of the performance of identification and rifampicin-resistance assays on primary specimens, and none in the context of a non-trial national clinical service. Sputum smear-positive cases of pulmonary tuberculosis are the most infectious and represent the most significant public health problem. For these reasons a rapid identification service for $M$. tuberculosis with the detection of rifampicin resistance primarily for patients with smear-positive sputum (called 'Fastrack') was initiated by the Public Health Laboratory Service Mycobacterium Reference U nit in December 1996. U rgent smear-negative sputum samples, broncho-alveolar lavages (BALs) and non-pulmonary specimens (apart from cerebrospinal fluids) were also accepted for testing by special request from clinicians during the same period and were analysed in parallel for completeness.

A clinical, microbiological and economic analysis of the 'Fastrack' service was performed to determine (1) clinical/epidemiological reasons underlying specimen referral; (2) concordance of molecular results with conventional identification and rifampicin drug susceptibility testing; (3) clinical and microbiological features of MDRTB cases; and (4) the potential economic impact of the service to NHS hospitals.

\section{M aterials and methods}

Clinical, microbiological and epidemiological data

Details of patient name, age, sex, ethnic group, specimen type, hospital, prior tuberculosis history, immunocompromised status, HIV positivity and clinical details (fever, weight loss, brief case history, chest radiography, treatment) were sought prospectively in all cases from the submitting clinician and laboratory. Details of additional risk factors including contact with patients with MDRTB, alcoholism or homelessness, non-compliance with treatment, other institutional residency (prison, residential home), and immigration and country of origin were determined retrospectively from the referring physician or by review of the medical notes. The significance of differences between patients with MDRTB and drug-sensitive tuberculosis was assessed by two-tailed Fisher's exact and unpaired $\mathrm{t}$ tests as appropriate.

\section{Processing of primary specimens and} identification of mycobacterial cultures

All experimental procedures were performed in a Class 1 Biological Safety Cabinet under Category 3 biohazard containment conditions. Sputum was stained with auramine-phenol and positive smears were confirmed by Ziehl-N eelsen staining [18]. Samples were decontaminated by the $\mathrm{NaOH} / \mathrm{NALC}$ method [18]. DNA was extracted from $1 \mathrm{ml}$ of decontaminated specimen [17]. The remaining specimen was used to inoculate an MB /BacT rapid culture vial (Organon Teknika, Durham, NC, USA) and one Lowenstein-Jensen solidmedium slope which was incubated for at least 8 weeks or 12 weeks if the specimen was from an HIV-positive patient.

Mycobacterial cultures from primary specimens submitted to the 'Fastrack' service were identified by microscopic and macroscopic appearance, biochemical tests, DNA hybridisation (A ccuprobe, Gen-Probe, San Diego, CA, USA) and thin-layer chromatography [18].

\section{Drug susceptibility testing}

The drug susceptibility of $M$. tuberculosis isolates was determined by the standard resistance ratio [18], radiometric BACTEC proportion (breakpoint concentration $=2 \mathrm{mg} / \mathrm{L}$ ) [19] and microbroth dilution MIC methods [20] and these were compared with the results from molecular assays.

Preparation of DNA extracts, PCR amplification and molecular rifampicin resistance assay

A nested PCR was performed with $10 \mu$ l of extracted DNA as template and with the outer LiPA primers (LiPA 1, 5'-GAGAATTCGGTCGGCGAGCTGATCC3'; LiPA2, 5'-CGAAGCTTGACCCGCGCGTACA (C $-3^{\prime}$ ) for the first round of amplification: 30 cycles of $95^{\circ} \mathrm{C}$ for $60 \mathrm{~s}, 58^{\circ} \mathrm{C}$ for $30 \mathrm{~s}$ and $72^{\circ} \mathrm{C}$ for $90 \mathrm{~s}$. One $\mu \mathrm{l}$ of the first round product was transferred to a $40-\mu \mathrm{l}$ reaction mixture containing the inner LiPA primers (LiPA 3, 5'-GGTCGGCATGTCGCGGATGG-3'; LiPA 4, GCACGTCGCGGACCTCCAGC) for the second round of amplification: $30 \mathrm{cycles}$ of $95^{\circ} \mathrm{C}$ for $20 \mathrm{~s}, 65^{\circ} \mathrm{C}$ for $30 \mathrm{~s}$ and $72^{\circ} \mathrm{C}$ for $30 \mathrm{~s}$ [14]. The inner primers were 
biotinylated at the $5^{\prime}$ end. Each reaction mixture contained $0.2 \mathrm{mmol}$ each of dATP, dCTP, dGTP, and $0.1 \mathrm{mmol}$ each of dTTP and dUTP, $20 \mathrm{pmol}$ of each primer and 1 unit of BIOTAQ DNA polymerase (Bioline, London). The Line Probe Assay (INNO-LiPA Rif.Tb; Innogenetics, Belgium) was used for the molecular rifampicin resistance test as described previously [14]. Extensive precautions, including the use of a three-room PCR suite, dedicated equipment and multiple negative controls were taken to avoid cross-contamination. High and low positive M. tuberculosis (c. 1000 and 100 genome copies) controls were included in parallel with patient samples.

\section{Economic analysis}

The time taken for routine culture, identification and susceptibility testing was determined for comparison with molecular identification and rifampicin resistance detection in all cases. The cost of inpatient bed stays and outpatient consultation visits was obtained [21]. Treatment costs were estimated conservatively as follows: daily combination formulations and six, monthly outpatient (OPD) visits for patients with drug-sensitive tuberculosis; the NHS billable cost of daily treatment for a minimum of 18 months for patients with MDRTB were calculated [3, 4, 22-24] assuming at least one inpatient stay of up to 56 days plus 18 OPD visits. Inpatient costs are billed as flat rates for each period of 28 days.

A more detailed analysis of the effect of rapid molecular rifampicin assays on bed usage at one London hospital was determined by review of bed occupancy data, billable costs and medical notes. The total bed days associated with occupation of negativepressure rooms between 1 A pril 1997 and 31 March 1998 was calculated. The excess days and costs associated with patients subsequently shown to have drug-sensitive tuberculosis but who were inappropriately isolated in negative-pressure rooms were calculated.

\section{Results}

\section{Clinical, microbiology and molecular results}

Rapid diagnosis of tuberculosis and detection of rifampicin resistance were performed on smear-positive sputum samples from 55 patients. The clinical, demographic and microbiological characteristics of all sputum smear-positive patients are given in Table 1. Of factors previously associated with drug resistance, 32 patients had a previous diagnosis of tuberculosis, five were HIV-positive, 21 were failing to improve on therapy, two were prisoners, two were contacts of M DRTB cases and 12 were immigrants from countries with a high incidence of tuberculosis overall or of resistant $M$. tuberculosis. Clinical data were reported on $43(78.1 \%)$ of the smear-positive patients. In 33 $(76.7 \%)$ of these cases, at least one of the following symptoms or signs was reported: fever, cough, weight loss, night sweats, haemoptysis, lymphadenopathy or a strong $M$ antoux reaction with an abnormal chest radiograph or two of the signs or symptoms alone. MDRTB was grown from sputum specimens from nine patients (eight smear-positive, one smear-negative). Overall, specimens from c. $1.7 \%$ of all bacteriologically confirmed cases in the UK and $1 \%$ of all reported notifications nationally were examined by the molecular rifampicin resistance test.

\section{Molecular identification results and factors associated with MDRTB}

The comparison of rapid tuberculosis and rifampicin resistance results with conventional identification and drug susceptibility testing is shown in Table 2. The concordance of molecular tuberculosis diagnosis and rifampicin resistance determination with conventional methods was $94.5 \%$ and $91.7 \%$, respectively.

Although the 'Fastrack' molecular rifampicin resistance service is specifically targeted to smear-positive sputum samples, clinicians submitted other specimens by special request. The concordance with tuberculosis diagnosis for smear-negative sputum samples was only

Table 1. Clinical, demographic and microbiological characteristics of all patients from whom smear-positive sputum samples were received $(n=55)$ by the 'Fastrack' molecular rifampicin resistance service

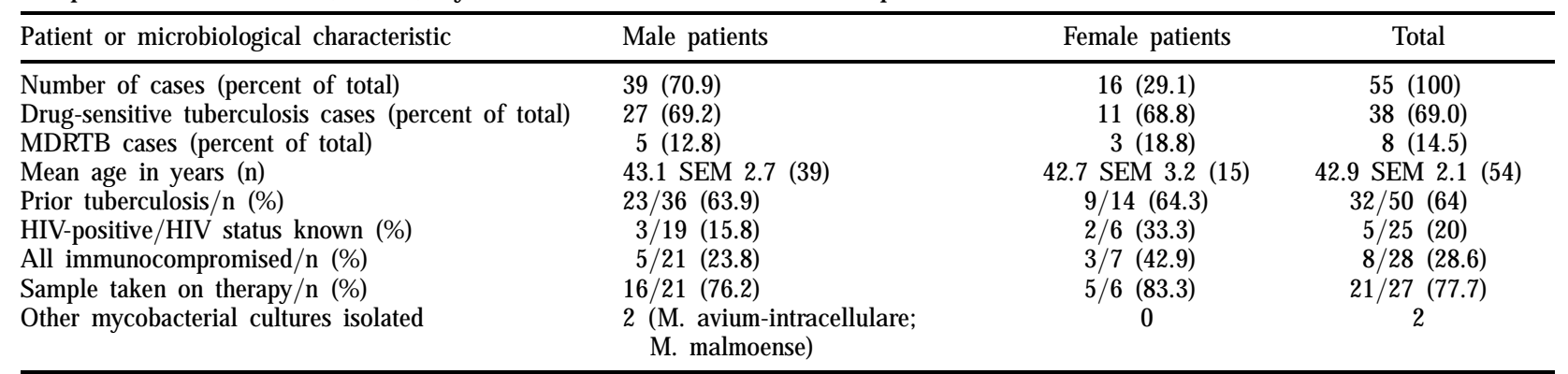

n, number of cases for which data were known. 
Table 2. 'Fastrack' molecular tuberculosis identification and rifampicin detection results

\begin{tabular}{|c|c|c|c|c|c|}
\hline Specimen & $\begin{array}{c}\text { Tuberculosis identification: } \\
\text { number correct/total } \\
\text { examined }\end{array}$ & $\begin{array}{c}\text { Concordance } \\
(\%)\end{array}$ & $\begin{array}{l}\text { Rifampicin resistance: } \\
\text { number correct/total } \\
\text { examined }\end{array}$ & $\begin{array}{c}\text { Concordance } \\
(\%)\end{array}$ & M ean days saved* \\
\hline $\begin{array}{l}\text { All primary specimens } \\
\text { Smear-positive sputum }\end{array}$ & $\begin{array}{l}81 / 91 \\
52 / 55\end{array}$ & $\begin{array}{l}89.0 \\
94.5\end{array}$ & $\begin{array}{l}55 / 61 \\
44 / 48\end{array}$ & $\begin{array}{l}90.2 \\
91.7\end{array}$ & 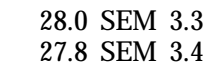 \\
\hline
\end{tabular}

$70 \%$ (7 of 10 ), but was $80.6 \%$ ( 29 of 36 ) for all sputum specimens, $84.6 \%$ ( 11 of 13 ) for BALs, $83.3 \%$ (5 of 6 ) for pleural fluids, $100 \%$ (2 of 2) for lymph nodes and $80.0 \%$ (4 of 5 ) for biopsies. For rifampicin resistance the concordance was only $50 \%$ (2 of 4) for smearnegative sputa, but was $80 \%$ (4 of 5) for BALs, $100 \%$ (2 of 2) for pleural fluids and 100\% (1 of 1) for lymph node.

Microbiological, clinical and risk factors underlying MDRTB cases

Table 3 summarises the principal clinical details of all nine (eight smear-positive, one smear-negative) MDRTB cases. All MDRTB cases had a previous

Table 3. Clinical and microbiological data on all MDRTB cases $(n=9)$

\begin{tabular}{lc}
\hline Clinical or microbiological factor & $\begin{array}{c}\text { Number }(\%) \text { of cases } \\
\text { with factor/number of } \\
\text { cases for which data } \\
\text { were known }\end{array}$ \\
\hline Male gender & $6 / 9(66.7)$ \\
Previous tuberculosis & $9 / 9(100)$ \\
Sample taken on therapy & $7 / 9(77.8)$ \\
Born overseas & $7 / 9(77.8)$ \\
HIV-positive & $1 / 8(12.5)$ \\
MDRTB (isoniazid + rifampicin only) & $4 / 9(44.4)$ \\
M DRTB (3 drugs) & $1 / 9(11.1)$ \\
MDRTB (4+ drugs) & $4 / 9(44.4)$ \\
Non-compliant & $4 / 9(44.4)$ \\
Alive at 6 months & $9 / 9(100)$ \\
\hline
\end{tabular}

diagnosis of tuberculosis and $>75 \%$ were born overseas. Nearly half were known to be non-compliant with therapy. Only one case was HIV-positive of eight cases in which the HIV status was known. Isolates from $>50 \%$ of the MDRTB cases were resistant to three or more drugs and one was resistant to seven drugs.

Table 4 compares potential microbiological and epidemiological risk factors between patients with cultureproven MDRTB and drug-sensitive tuberculosis in sputum smear-positive cases. More smear-positive sputum samples came from men $(70.9 \%)$, although there was no significant difference between the proportion of males among MDRTB and drug-sensitive tuberculosis cases. The number of cases born overseas in the MDRTB and drug-sensitive tuberculosis groups was significantly different, but was more likely to have been reported in the MDRTB group. No significant difference was seen in the proportion of HIV-positive or immunocompromised individuals amongst patients with MDRTB or drug-sensitive tuberculosis, although HIV status was known in only $54 \%$ of smear-positive cases. It was more likely to be known in the MDRTB group.

The time taken to culture, identify and determine rifampicin resistance (and resistance to other first-line drugs) in samples from all MDRTB cases was significantly longer than for all drug-sensitive tuberculosis cases overall (mean $=55$ SEM 14.8 days versus 31 SEM 2.5 days; $\mathrm{t}=2.83$, $\mathrm{df}=44$ ). There was no

Table 4. Microbiological and clinical risk factors associated with smear-positive cases

\begin{tabular}{|c|c|c|c|}
\hline \multirow[b]{2}{*}{ Factor } & \multicolumn{2}{|c|}{$\begin{array}{c}\text { Number with factor/number of cases in } \\
\text { which data are known (\%) }\end{array}$} & \multirow[b]{2}{*}{ Significant difference ( $p$ value) } \\
\hline & MDRTB cases & $\begin{array}{l}\text { Drug-sensitive } \\
\quad \text { cases }\end{array}$ & \\
\hline $\begin{array}{l}\text { Male sex } \\
\text { Prior tuberculosis }\end{array}$ & $\begin{array}{l}5 / 8(62.5) \\
8 / 8(100)\end{array}$ & $\begin{array}{l}28 / 38(73.7) \\
19 / 34(55.9)\end{array}$ & $\begin{array}{l}\text { No }(0.6689) \\
\text { Yes }(0.0352) \\
O R=13.51(\mathrm{Cl} 0.722-253.0)\end{array}$ \\
\hline $\begin{array}{l}\text { HIV-positive } \\
\text { Born overseas }\end{array}$ & $\begin{array}{l}1 / 7(14.3) \\
7 / 8(87.5)\end{array}$ & $\begin{array}{l}3 / 15(20.0) \\
5 / 23(21.7)\end{array}$ & $\begin{array}{l}\text { No }(1.000) \\
\text { Yes }(0.002) \\
\text { OR }=25.2(\mathrm{Cl} 2.5-256)\end{array}$ \\
\hline $\begin{array}{l}\text { A ge } \\
\text { Samples taken while on therapy } \\
\text { Successful cultures from samples taken on therapy } \\
\text { Days to culture, TB ID\&R } \\
\text { Days to culture, TB ID\&R of samples taken on therapy }\end{array}$ & $\begin{array}{l}5 \text { SD } 4.0 \\
6 / 8(75) \\
6 / 7(85.7) \\
55 \text { SEM } 14.8(8)^{*} \\
47.5 \text { SEM } 14.3(6)\end{array}$ & $\begin{array}{l}45.4 \text { SD } 2.6 \\
16 / 38(42.1) \\
11 / 16(68.8) \\
31 \text { SEM } 2.5(38) \\
36 \text { SEM } 5.1(11)\end{array}$ & $\begin{array}{l}\text { No }(t=1.726 ; d f=44) \\
\text { No }(0.1278) \\
\text { No }(0.6214) \\
\text { Yes }(t=2.827 ; d f=44) \\
\text { No }(t=0.927 ; d f=15)\end{array}$ \\
\hline
\end{tabular}

*8 MDRTB, 38 drug-sensitive cases; TB ID\&R, days taken to culture, identify M. tuberculosis and determine rifampicin determination in all specimens or in samples known to have been taken on therapy. 
significant difference between the days taken to culture and identify MDRTB and drug-sensitive tuberculosis cases (mean $=47.5$ days SEM 14.3 days versus 36 SEM 5.1 days; $t=0.928 ; d f=15$ ) when samples from cases known to be on therapy were considered. The number of days taken to culture specimens from MDRTB patients on therapy appears shorter than the time taken for the whole MDRTB group, but although the treatment status of $25 \%$ of the MDRTB patients was not defined it is highly likely that they were also on supervised chemotherapy at the time the sample was taken.

\section{Discrepant results}

Table 5 lists the discrepant rifampicin resistance results, i.e., those individuals from whom sputum was obtained in which the molecular rifampicin resistance result did not agree with that from the conventional assay.

In case I, the rapid molecular assay correctly identified the presence of $M$. tuberculosis in the sputum but did not detect mutations associated with rifampicin resistance. The resulting culture was also correctly identified as M. tuberculosis, but was rifampicin-sensitive by molecular assay. Conventional culture-based drug susceptibility testing showed the isolate to be low-level resistant at two reference centres but sensitive at a third. The patient was from Eastern Europe, gave no prior history of tuberculosis and had significant cavitatory disease. He was cured on quadruple therapy supplemented with additional agents.

In case II, DNA sequencing confirmed the presence of a mutation that would normally confer resistance (data not shown). However, testing by the resistance ratio, radiometric BACTEC and microbroth dilution MIC assays indicated rifampicin sensitivity. As mutations were clearly present it is possible that compensatory mutations elsewhere in the gene restored at least partial function.

Case IV was a male diabetic who had been admitted with drug-sensitive tuberculosis in 1996 and later admitted to the same institution as a relapsed case in 1997. Isoniazid resistance was identified in 1997 and the patient was subsequently admitted for directly observed therapy because of poor compliance. Although rifampicin resistance was identified on conventional testing, a low number of colonies was seen, i.e., borderline rifampicin resistance. Subsequently, restriction fragment-length polymorphism analysis with the insertion sequence IS6110 indicated that this isolate and that from case $V$ were the same (data not shown), i.e., undiagnosed nosocomial transmission had occurred. The sputum and isolate in case $\mathrm{V}$ gave the same results on molecular analysis as for case IV and came from a second individual at the same institution. This patient was also a male with an isoniazid-resistant isolate identified 3 months previously who was admitted for non-compliance with his therapy.

\section{Economic analysis}

An analysis was performed of the additional costs associated with case management in negative-pressure room isolation of potential MDRTB cases over 1 year at one major London hospital (Table 6). Between 1 A pril 1997 and 31 March 1998, there were 15 patient episodes involving isolation in negative-pressure rooms for a total of 449 days. Two HIV-positive patients had MDRTB and were isolated appropriately for 96 days, indicating that 353 days of negative-pressure-based care was used inappropriately. If it is assumed, conservatively, that for each episode it would have taken 5 days to identify tuberculosis and determine rifampicin susceptibility by molecular analysis, then 75 days $(5 \times 15)$ would be subtracted from the total leaving 278 days of 'wasted extra cost'. The savings that one institution, albeit in an area of high TB incidence, might make was a maximum of $278 \times f 552=f 153456$. The floor area occupied by a negative-pressure room for one patient at this institution is approximately twice that of a conventional thoracic bed, so it might be considered that the minimum saving would be that of the 'extra bed' or $£ 198$ per day $=£ 55044$. Therefore, one hospital could have saved between $£ 50000$ and $£ 150000$ (Table 6).

\section{Discussion}

A national molecular service for the identification of $M$. tuberculosis and the detection of rifampicin resistance in smear-positive sputum was instituted after

Table 5. A nalysis of discrepant rifampicin-resistant results in sputum samples compared to conventional analysis

\begin{tabular}{ll}
\hline Specimen type & Case number and notes \\
\hline Smear-positive sputum & I. Culture and sputum examined; conflicting conventional rifampicin result (see text) \\
& II. Resistant tuberculosis by rapid assay, sensitive by conventional assay. Previous therapy, poor compliance; \\
recovery on quadruple therapy (see text) & III. Sensitive tuberculosis by rapid assay, resistant by conventional assay; M DRTB isolate \\
& IV. Sensitive tuberculosis by rapid assay, resistant by conventional assay; M DRTB; this isolate and that from case \\
& V were the same, i.e., nosocomial transmission (see text) \\
Smear-negative sputum & V. Sensitive tuberculosis by rapid assay, resistant by conventional assay; linked to case IV. Subsequent culture had \\
& S2 band mutation by InnoLipa, i.e., rifampicin-resistant \\
& VI. Resistant tuberculosis by rapid assay, sensitive tuberculosis by conventional assay \\
\hline
\end{tabular}


Table 6. Potential annual savings at one London hospital by rapid identification of MDRTB cases and reduction in inappropriate negative-pressure room isolation

\begin{tabular}{ll}
\hline Number of inpatient isolation episodes in NPR rooms (1997-1998) & 15
\end{tabular}

Total number of NPR isolation days* 449 days

Number of 'correct' NPR isolation days for MDRTB (2 MDRTB cases)*

Inappropriate NPR isolation days (449-96), i.e., days in which drug-sensitive cases were isolated in NPR

Assume 5 days to rapidly identify $M$. tuberculosis and rifampicin resistance per episode $(15 \times 5)$

Final wasted drug-sensitive tuberculosis days spent in NPR (353-75)

Excess daily cost (NPR day cost - daily thoracic bed day cost) ${ }^{\dagger}(£ 750-198)$

(a) M aximum saving $(278 \times f 552)$

(b) Using 'extra bed' floor space calculation (see text) $(278 \times f 198)$

(c) Individual test costs for analysis of 2 specimens per NPR episode $(15 \times 2 \times f 120)$

(d) Final cost saving $(\mathrm{a}-\mathrm{c})$ or $(\mathrm{b}-\mathrm{c})$

(e) NHS costs for ambulatory care drug-sensitive cases $(70 \mathrm{~kg}$; daily quadruple therapy 2 months; 4 months $\mathrm{R}+\mathrm{I} ; 6$ OPD visits

(f) Minimum NHS billed MDRTB costs (up to 56 days as inpatient; $18 \mathrm{OPD}$; $\mathrm{Ci}+\mathrm{Pz}+\mathrm{E}$ daily therapy for 18 months ambulatory (are)

NPR, negative-pressure isolation; OPD, outpatient department; $R$, rifampicin; I, isoniazid; Ci, ciprofloxacin; Pz, pyrazinamide; $E$, ethambutol. *Isolation in accordance with published guidelines $[23,24]$.

${ }^{\dagger}$ Costs derived [22].

These are billed NHS inpatient ( $£ 2142$ up to 28 days) and outpatient ( $£ 156$ per visit) costs not actual daily costs plus drug costs [25] for three drugs for minimum 18 months therapy.

a pilot research study. Other primary specimens were examined during this period in response to clinical request.

Four studies in the USA $[3,4,25-27]$ had indicated a reduced mortality (even among HIV-positive individuals) as a result of early MDRTB detection and the institution of therapy based on in-vitro drug susceptibility testing. For example, in a study of 38 mainly HIV-positive MDRTB patients, an overall response rate of $50 \%$ was seen with a median survival of 315 days [25]. First-line drug susceptibilities were available in a median of 30 days. Therefore, the rationale for a national molecular rifampicin resistance service would be to improve patient survival by the early identification of MDRTB. This would lead to the immediate addition of further anti-tuberculosis drugs to the patient's regimen which would subsequently be tailored on the basis of in-vitro drug susceptibility testing. All nine MDRTB patients identified in this study were placed on additional drugs immediately and therapy was modified subsequently on the basis of in-vitro drug susceptibility testing. All survived at least 6 months of follow-up.

M ore samples were taken while the patient was on drug therapy among MDRTB cases than drug-sensitive cases, but this was not statistically significant. Where declared, $>75 \%$ of smear-positive sputum samples were taken while the patient was on therapy. Conventionally, the culture of samples taken from patients on therapy is believed to be of little value, particularly in drug-sensitive cases, as bacteria would not be viable. $\mathrm{Nevertheless,} \mathrm{culture} \mathrm{was} \mathrm{successful} \mathrm{in} \mathrm{samples} \mathrm{taken}$ from 11 patients with drug-sensitive tuberculosis as well as MDRTB cases on therapy. There were several features in the group of patients with drug-sensitive tuberculosis that indicated compliance problems that would make successful culture more likely: seven had had a prior diagnosis of tuberculosis, nine had failed to improve on therapy, three were alcoholic and six were non-compliant with treatment, two of whom were subsequently detained in hospital to improve compliance.

In general, culture is more likely to be successful in those patients taking no medication, taking their medication erratically or in drug-resistant cases, but using the most advanced culture systems it is possible to obtain positive cultures from many samples taken on therapy, as shown here.

Rapid identification of tuberculosis in smear-positive specimens has been performed by different molecular amplification techniques. There are clear benefits in also determining rifampicin resistance and a survey of UK clinical laboratories indicated that a national service to determine resistance in smear-positive sputum samples was needed [28].

In this study, results were compared with the prospectively determined 'gold standard' of bacteriological culture even where there were clear reasons for discrepant results. 'False negatives' can be explained in that amplification-based assays are not as sensitive as culture methods, which is supported here in that only $70 \%$ of smear-negative culture-positive sputum samples were identified as tuberculosis, which is in line with other published studies. 'False-positives' (i.e., PCR products with a negative culture) were probably due to specimens which were taken whilst the patient was on treatment or loss of viability during specimen decontamination. For example, cases IV and V were on chemotherapy when samples were taken. The decontamination process accounts for the inability to culture a small percentage of smear-positive sputum specimens and laboratories aim to limit the loss of viable cultures through the decontamination process to c. $2 \%$.

All the sputum specimens for which there were discrepant rifampicin-resistant results were correctly identified as M. tuberculosis. Most discrepant results 
were due to low-level resistance isolates. Interestingly, a single MDRTB type was demonstrated by IS6110 DNA fingerprinting (data not shown) to be responsible for cases IV and V, identifying a small, previously unknown, nosocomial tuberculosis outbreak.

A gainst these encouraging results, between $5 \%$ and $10 \%$ of genuine rifampicin resistance might not be detected by this genotypic method $[11,15,17]$. It is likely that the full cost savings could not be achieved until the accuracy of these methods can be improved. Although the cost-effectiveness of rapid molecular tuberculosis identification systems has been debated [29-31], there has been limited analysis of the likely benefits of a molecular rifampicin resistance service in the context of a state-funded health care service. The A merican Thoracic Society consensus statement on rapid diagnostic tests for the diagnosis of tuberculosis indicated that, although these tests had been evaluated in clinical laboratories, they had not been examined for their utility in 'routine clinical use or public health settings' [31].

In the UK, mycobacterial cultures are identified and drug resistance determined by the Public Health Laboratory Service (PHLS) and Scottish Mycobacteria R eference $L$ aboratory at no cost to hospital laboratories, but the examination of primary samples will in general incur a charge. In practice, therefore, those requesting molecular rifampicin resistance tests would have made an informal cost-benefit analysis based on the charge for the test and potential costs saved or clinical benefits accrued by an earlier result. During this period, 9 (9.9\%) of 91 specimens referred for rapid rifampicin resistance analysis by clinicians were MDRTB, indicating that specimens were being selected successfully on the basis of putative risk factors, because the UK national M DRTB incidence rate was $<2 \%$ [10].

A practical consensus might consider three costeffective areas of use for molecular diagnosis in tuberculosis: (1) the identification of infections as M. tuberculosis and not $M$. avium-intracellulare (MAI) in immunocompromised patients where either diagnosis is likely; (2) the diagnosis of drug-sensitive tuberculosis (or MDRTB) where the consequences of a missed diagnosis would be severe as in tuberculosis meningitis; and (3) to determine appropriate levels of patient isolation. Institutions would benefit as no infection control measures would be necessary if the 'smearpositive case' was due to infection with MAl rather than M. tuberculosis. Similarly, high costs are incurred in treating potential MDRTB patients in accordance with UK guidelines [22, 23], which require treatment in negative-pressure isolation facilities; rapid analysis would be valuable in determining the need for costly negative-pressure isolation. M olecular rifampicin resistance assays can reduce the excess costs associated with the isolation of patients with risk factors for MDRTB who subsequently are shown to have drug- sensitive tuberculosis, as these patients could be treated in cheaper ward-based side-rooms. Similarly the scope of contact tracing will be influenced by these tests.

The actual test costs included rapid culture and the numerous controls essential to the identification of cross-contamination and the maintenance of an accurate quality service. Nevertheless, significant institutional savings could be made. The cost of molecular rifampicin assays represents $<10 \%$ and $1 \%$ of the total NHS billing cost (i.e., the cost that the NHS uses in extracontractual referrals to other NHS hospitals rather than the true 'real' cost, which is higher) of ambulatory treatment for drug-sensitive tuberculosis and for MDRTB treatment, respectively, in the UK (Table 6).

The high infrastructure costs needed to perform molecular rifampicin resistance assays reliably with the ability to culture isolates, confirm molecular rifampicin resistance and identify susceptibility to other anti-tuberculosis drugs would support a centralised model to reduce the unit cost of each test [32], in the context of a country with a low incidence of rifampicin resistance and MDRTB. A dequate staffing and infrastructure with the use of multiple appropriate controls to identify cross-contamination are essential, as attempts to perform these assays without proper facilities and safeguards leads to significant error. Noordhoek et al. [33] sent 200 BCG-spiked specimens as part of a blinded study to seven laboratories which were skilled in PCR-based detection methods. All laboratories were able to detect $10^{7}$ organisms $/ \mathrm{ml}$, but only two were able to detect $10^{4}$ organisms $/ \mathrm{ml}$. False positive rates of 0 $77 \%$ were detected. The best results were obtained when PCR-based methods were performed in large clinical laboratories with sufficient space to separate preparation and detection areas, with the ability to culture specimens to audit PCR performance and to perform DNA fingerprinting to detect contamination.

Clinically, early diagnosis of rifampicin resistance and MDRTB permits the early instigation of appropriate therapy, as discussed earlier. Nevertheless, in the UK, 380000 specimens are taken annually from patients in whom tuberculosis is a differential diagnosis, but they yield only c. 3500 bacteriologically proven cases $[10,28]$. Therefore, use of these tests for screening would be inappropriate, but it would be appropriate to apply these tests to infectious patients with risk factors for drug resistance including contact with MDRTB cases, patients with prior tuberculosis and those born overseas in high incidence countries, as well as at risk immunocompromised individuals.

Nationally, the savings that might be made would depend on the local incidence of tuberculosis and M DRTB, in particular, and the availability of negativepressure isolation. There are 425 NHS [34] provider hospital trusts in England and Wales and if it is assumed that a single day of negative-pressure isolation 
was saved by each institution, the cost of a centrally organised service would be provided for.

The performance of molecular rifampicin resistance assays is technically feasible, and in a low-incidence country with adequate transport infrastructure, a centralised model is an effective one. A reas such as London with a higher incidence of tuberculosis and a higher rate of drug resistance can benefit from a central model targeted at the most infectious cases and those which are likely to be rifampicin-resistant (including samples from patients with prior tuberculosis, birth in high incidence countries, contact with MDRTB cases and poor compliance). In this, smear-positive specimens are referred to a single centre for rapid culture and analysis of rifampicin and isoniazid resistance with molecular rifampicin assays applied to those specimens with risk factors for drug resistance as described above [32].

We thank Mr M. Yates for performing the microtitration plate drug susceptibility assays and for advice on interpretation of the resistance ratio susceptibility results. We also thank all laboratory directors and clinicians for their assistance.

\section{References}

1. Drobniewski F, Pablos-M endes A, Raviglione MC. Epidemiology of tuberculosis in the world. Semin Respir Crit Care Med 1997; 18: 419-429.

2. Pablos-Méndez A, Raviglione MC, Laszlo A et al. for the World Health Organization-International Union Against Tuberculosis and Lung Disease Working Group on AntiTuberculosis Drug resistance Surveillance. Global surveillance for antituberculosis-drug resistance, 1994-1997. N Engl J Med 1998; 338: 1641-1649.

3. Iseman MD. Treatment of multidrug-resistant tuberculosis. N Engl J Med 1993; 329: 784-791.

4. Drobniewski FA. Is death inevitable with multi resistant TB plus HIV infection? Lancet 1997; 349: 71-72.

5. Edlin BR, Tokars JI, Grieco MH et al. An outbreak of multidrug resistant tuberculosis among hospitalized patients with the acquired immunodeficiency syndrome. N Engl J Med 1992; 326: $1514-1521$.

6. Small PM, Shafer RW, Hopewell PC et al. Exogenous reinfection with multidrug-resistant $M$ ycobacterium tuberculosis in patients with advanced HIV infection. N Engl J Med 1993; 328: 1137-1144.

7. Breathnach AS, de Ruiter A, Holdsworth GMC et al. An outbreak of multi-drug resistant tuberculosis in a London teaching hospital. J Hosp Infect 1998; 39: 111-117.

8. Goble M, Iseman MD, Madsen LA, Waite D, Ackerson L, Horsburgh CR. Treatment of 171 patients with pulmonary tuberculosis resistant to isoniazid and rifampin. N Engl J Med 1993; 328: 527-532.

9. Ormerod LP, Charlett A, Gilham C, Darbyshire JH, Watson JM. Geographical distribution of tuberculosis notifications in national surveys of England and Wales in 1988 and 1993: report of the Public Health Laboratory Service/B ritish Thoracic Society/Department of Health Collaborative Group. Thorax 1998; 53: 176- 181.

10. Irish C, Herbert J, Bennett D et al. Database study of antibiotic resistant tuberculosis in the U nited Kingdom 1994- 6 . BMJ 1999; 318: 497-498.

11. Telenti A, Imboden P, Marchesi $F$ et al. Detection of rifampicin-resistance mutations in Mycobacterium tuberculosis. Lancet 1993; 341: 647-650.

12. Heym B, Honoré $N$, Truffot-Pernot $C$ et al. Implications of multidrug resistance for the future of short-course chemotherapy of tuberculosis: a molecular study. Lancet 1994; 344: 293- 298.
13. Musser JM. Antimicrobial agent resistance in mycobacteria: molecular genetic insights. Clin Microbiol Rev 1995; 8: 496- 514.

14. De Beenhouwer $H$, L hiang $Z$, Jannes $G$ et al. Rapid detection of rifampicin resistance in sputum and biopsy specimens from tuberculosis patients by PCR and line probe assay. Tuber Lung Dis 1995; 76: 425-430.

15. Cooksey RC, Morlock GP, Glickman S, Crawford JT. Evaluation of a line probe assay kit for characterization of rpoB mutations in rifampin-resistant Mycobacterium tuberculosis isolates from New York City. J Clin Microbiol 1997; 35: 1281- 1283.

16. Nash KA, Gaytan A, Inderlied CB. Detection of rifampicin resistance in Mycobacterium tuberculosis by use of a rapid, simple, and specific RNA/RNA mismatch assay. I Infect Dis 1997; 176: 533- 536.

17. Watterson SA, Wilson SM, Yates MD, Drobniewski FA. Comparison of three molecular assays for rapid detection of rifampicin resistance in Mycobacterium tuberculosis. I Clin Microbiol 1998; 36: 1969-1973.

18. Collins $\mathrm{CH}, \mathrm{Grange} J \mathrm{M}$, Yates M D. Tuberculosis bacteriology: organization and practice, 2nd edn. Oxford, ButterworthHeinemann. 1997.

19. Heifets $L B$, Good RC. Current laboratory methods for the diagnosis of tuberculosis. In: Bloom BR (ed) Tuberculosis pathogenesis, protection and control. Washington, DC, ASM Press. 1994: 85- 110.

20. Telles MAS, Yates MD. Single and double drug susceptibility testing of Mycobacterium avium complex and mycobacteria other than the tubercle (MOTT) bacilli by a micro-dilution broth minimum inhibitory concentration (MIC) method. Tuber Lung Dis 1994; 75: 286-290.

21. NHS Executive. Tuberculosis control in London - the need for change. A report for the Thames Regional Directors of Public Health. London, NHS Executive. 1998.

22. Joint Tuberculosis Committee of the British Thoracic Society. Chemotherapy and management of tuberculosis in the United Kingdom: recommendations 1998. Thorax 1998; 53: 536-548.

23. Interdepartmental Working Group on Tuberculosis. UK Guidance on the prevention and control of transmission of HIVrelated tuberculosis, drug-resistant, including multiple drugresistant tuberculosis. London, UK Department of Health. 1998.

24. British National Formulary. British Medical Association and Royal Pharmaceutical Society of Great Britain. 1998: 35.

25. Turett GS, Telzak EE, Torian LV et al. Improved outcomes for patients with multidrug-resistant tuberculosis. Clin Infect Dis 1995; 21: 1238-1244.

26. Telzak EE, Sepkowitz K, Alpert $P$ et al. Multidrug-resistant tuberculosis in patients without HIV infection. N Engl J Med 1995; 333: 907-911.

27. Park MM, Davis AL, Schluger NW, Cohen $H$, Rom WN. Outcome of MDR-TB patients, 1983-1993: prolonged survival with appropriate therapy. Am J Respir Crit Care Med 1996; 153: 317- 324

28. Drobniewski FA, Watt B, Smith EG et al. A national audit of the laboratory diagnosis of tuberculosis and other mycobacterial diseases within the United Kingdom. J Clin Pathol 1999; 52: $334-337$.

29. Roth A, Schaberg T, Mauch H. Molecular diagnosis of tuberculosis: current clinical validity and future perspectives. Eur Respir J 1997; 10: 1877-1891.

30. Bennedsen J, Thomsen VO, Pfyffer GE et al. Utility of PCR in diagnosing pulmonary tuberculosis. J Clin Microbiol 1996; 34: 1407-1411.

31. The Workshop Report of the American Thoracic Society Workshop. Rapid diagnostic tests for tuberculosis - what is the appropriate use? Am J Respir Crit Care Med 1997; 155: 1804- 1814

32. Drobniewski F. Diagnosing multidrug resistant tuberculosis in B ritain. Clinical suspicion should drive rapid diagnosis. BMJ 1998; 317: 1263-1264.

33. Noordhoek GT, Kolk AHJ, Bjune G et al. Sensitivity and specificity of PCR for detection of Mycobacterium tuberculosis: a blind comparison study among seven laboratories. J Clin Microbiol 1994; 32: 277-284

34. Jewell T. United Kingdom - National health under new management. Lancet 1997; 350: 48-50. 